\title{
SOBRE ALGUNOS TRABAJOS \\ DE LOS FORMALISTAS RUSOS \\ EN SUS VERSIONES CASTELLANAS
}

José Domínguez Caparrós

Aquel que conozca la teoría literaria del formalismo ruso sólo a través de la traducción de sus trabajos al castellano - y esta suele ser la primera forma de acercamiento para muchos estudiantes- se encuentra con varios problemas. En primer lugar, descubre que de algunos trabajos hay dos traducciones distintas. Esto ocurre en el caso de las siguientes obras: Tynianov, Eikhenbaum y Shklovski: Formalismo y vanguardia. Textos de los formalistas rusos, vol. 1 . Traducción de Agustín García Tirado y Juan Antonio Méndez. Madrid, Alberto Corazón Editor, 1973, 2,a edición. Tres de los cuatro trabajos aquí recogidos están también en la traducción castellana de la famosa antología que publicó en francés Tzvetan Todorov. Los trabajos repetidos son: B. Eikhenbaum, «La teoría del "método formal"»; V. Sklovski, "El arte como procedimiento»; Y. Tinianov, "De la evolución literaria». El cuarto trabajo, perteneciente a Y. Tinianov y titulado "Sobre la composición del Eugeni Onegin», no se encuentra traducido en ningún otro sitio. Este trabajo estuvo inédito en ruso hasta 1967, según advierten los editores españoles. Notemos, de pasada, que los nombres propios son transcritos de una forma en la portada del libro, y de forma distinta en el interior. Los traductores de los trabajos aquí reunidos no dicen de qué lengua traducen, aunque el castellano empleado en la traducción publicada en Madrid es más legible que el de la traducción de la editorial Siglo XXI.

En la traducción castellana de la antología de textos de los formalistas rusos traducidos al francés por $\mathrm{T}$. Todorov hay otros trabajos - de R. Jakobson y de B. Tomachevski- que es posible encontrar en otras traducciones castellanas. Primero, la referencia bibliográfica de la traducción castellana de la antología de T. To- 
dorov: Teoria de la literatura de los formalistas rusos, por Jakobson, Tinianov, Eichenbaum, Brik, Shklovski, Vinogradov, Tomashevski, Propp. Antología preparada y presentada por Tzvetan Todorov. Traducción de Ana María Nethol. México, Siglo XXI, 1980, 4.a edición. La 1. ${ }^{\text {a }}$ edición de esta obra se publicó en Buenos Aires, Ediciones Signos, 1970. (De ahora en adelante abrevio en $T$. L. la referencia a esta obra.) El trabajo de Roman Jakobson titulado «Sobre el realismo artístico" —en $T$. L., págs. 71-79 - se encuentra también traducido en la obra colectiva (George Lukacs, T. W. Adorno, Roman Jakobson, Ernst Fisher, Roland Barthes) Polémica sobre realismo, Buenos Aires, Editorial Tiempo Contemporáneo, 1972, 2. ${ }^{\text {a }}$ edición (1. edición: 1969). El título del trabajo en esta colección, donde ocupa las páginas 157-176, es «El realismo artísticon, y lo traduce -no dice de qué lengua- Floreal Mazza.

El trabajo de B. Tomashevski, «Temática» (en $T$. L., págs. 199-232), es un fragmento de una obra más amplia de este autor que ha sido traducida completa al castellano recientemente. Se trata de Boris Tomachevski, Teoría de la literatura. Prólogo de Fernando Lázaro Carreter. Traducción de Marcial Suárez. Madrid, Akal editor, 1982. No dice el traductor de qué lengua traduce, aunque da el título de la obra en ruso y la fecha de su aparición (1928), que no se corresponde con la fecha que dan V. Erlich y T. Todorov para la 1. edición de esta obra (1925). El fragmento recogido en $T$. $L$. corresponde a las páginas $179-215$ de la traducción completa de la obra de Tomachevski. Es preferible leer el trabajo de Tomachevski en esta traducción completa.

Uno de los fragmentos de Tinianov publicado en T.L. (páginas 85-88) con el título de «La noción de construcción» se encuentra traducido también al castellano en la edición de la obra de Tinianov a que pertenece dicho fragmento. Se trata de El problema de la lengua poética. Traducción de Ana Luisa Poljak. Buenos Aires, Siglo XXI, 1972. No se dice de qué lengua traduce, aunque, al dar el título de la obra en ruso y el título de su versión italiana, parece deducirse que se ha traducido del italiano. El fragmento incluido en $T$. $L$. corresponde a las páginas 11-14 de la traducción completa.

Una segunda cuestión sería la de establecer el corpus de trabajos formalistas asequibles en traducciones castellanas. Tal corpus esencial estaría compuesto por los siguientes títulos:

1. Teoría de la literatura de los formalistas rusos, ya citada.

2. Tinianov, Iuri: El problema de la lengua poética, ya citada.

3. Jakobson, Roman: «El folklore como forma específica de creación*, en Ensayos de Poética. Traducción de Juan Almela. 
Madrid, F.C.E., 1977, páginas 7-22. El caso de este libro merece también un pequeño comentario. Se da como título original de esta obra en francés el de Questions de Poétique. Paris, Editions du Seuil, 1973, y, sin ningún aviso, resulta que la traducción castellana no recoge más que once de los veintinueve textos publicados en la edición francesa que dirigió $T$. Todorov y que recoge cinco textos más de la época formalista de Roman Jakobson.

4. Tomachevski, Boris: Teoría de la literatura, ya citada.

5. Propp, Vladimir: Morfología del cuento. Traducción de Lourdes Ortiz. Madrid, Editorial Fundamentos, 1974, 2." edición. Al principio de la obra hay una nota del traductor -que debería servir de modelo para las otras traducciones- referida a la edición y lengua de la que se traduce. Es traducción del francés. La misma Editorial Fundamentos ha publicado otros trabajos de V. Propp posteriores a la época formalista. Entre ellos se puede citar Las raíces históricas del cuento, obra de 1946.

Dejo fuera la referencia a algunos trabajos de V. Sklovski publicados por las editoriales Planeta y Anagrama. La razón es que o no son trabajos de teoría literaria o son trabajos posteriores a la etapa formalista. Con todo, en ellos se pueden encontrar frecuentes alusiones al movimiento formalista y su pensamiento. Algunos títulos: Sobre la prosa literaria, La cuerda del arco, Zoo o cartas no de amor, Viaje sentimental, Crónicas de la revolución rusa, etc.

Una vez establecido el corpus de trabajos de los formalistas disponibles en traducción al castellano - de los que, como hemos visto, algunos están traducidos dos veces, y de otros no se dice de qué lengua se traduce--, se plantea una tercera cuestión: la de la calidad y fiabilidad de dichas traducciones. Aunque yo no soy traductor, ni conozco, desgraciadamente, la lengua rusa, sí hay algunos detalles que quisiera comentar. Pues basta con conocer la edición francesa más importante de textos de los formalistas rusos para darse cuenta de la precaución que hay que tener a la hora de manejar la traducción castellana de tal antología. En efecto, me voy a limitar a unas cuantas observaciones sobre la traducción de la antología de Todorov, que constituye la fuente clásica para el conocimiento de las teorías formalistas en nuestro ámbito cultural.

Mi primera observación se va a referir a la manera arbitraria en que se han manejado las notas. En esta línea puedo citar la supresión de algunas notas que hay en el texto francés. (De ahora en adelante abrevio en $T h L$ la referencia al texto francés y sigo refi- 
riéndome al texto español con la abreviatura $T$. $L$.; el número indica la página.) Véase: $T h L, 54$, y $T . L ., 38 ; T h L, 84$, y T.L., 61; ThL, 140, y T.L., 104; ThL, 178, y T.L., 133; ThL, 180, y T.L., 135; ThL, 196, y $T . L ., 146 ; T h L, 223$, y $T . L ., 167$. Hay, igualmente, algunos casos de notas del traductor español que no están en el original francés y que tampoco llevan la indicación de que sean notas del traductor. Véase: $T h L, 108$, y $T . L ., 79 ; T h L, 109$, y $T . L ., 81 ; T h L, 191$, y $T . L ., 143$. En algún caso se apropia una nota mediante la indicación N. del T., cuando se ha limitado a cambiar algo la nota de la edición francesa $(T h L, 184$, y $T . L ., 137)$, o simplemente da como propia una nota del autor ruso traducido $(T h L, 161$, y $T . L ., 120)$.

La segunda observación se refiere a la supresión de parte del material y noticias que da al final de la traducción la edición francesa. Así, se suprime, sin aviso previo, la parte final de la nota bibliográfica en que el editor francés se refiere a otras traducciones de textos formalistas a lenguas occidentales, al libro de V. Erlich como fuente de información más completa sobre el formalismo, o a reimpresiones de textos formalistas rusos en Occidente. Tampoco son de fiar las notas sobre los autores. Así, en el caso de Sklovski, suprime la referencia a la traducción al francés de dos libros suyos de los años veinte (Viaje sentimental y Zoo), libros que también están traducidos al castellano. Suprime, igualmente, la referencia a la aparición en francés de los Ensayos de lingüística general, de $R$. Jakobson. Tampoco se comprende que el traductor añada al final de la nota sobre V. V. Vinogradov: "Actualmente está a la cabeza de los linguiistas soviéticos.» Suprime también el índice de materias que viene al final de la edición francesa.

La tercera observación que voy a hacer se refiere a un problema que el traductor no resuelve de forma satisfactoria. Se trata del problema de la traducción del término francés nouvelle, que, como es sabido, se corresponde con el de novela corta. (Sigo, en este punto, la opinión de Carlos García Gual, quien plantea el problema de la terminología en las lenguas europeas occidentales para los distintos géneros narrativos, y adopta el término de novela corta para traducir el francés nouvelle. V. "Relaciones entre la novela corta y la novela en la literatura griega y latina», Faventia, 1/2, 1979, U. A. Barcelona, págs. 135-153.) Pues bien, la solución que adopta el traductor cada vez que aparece en el texto francés el término nouvelle no puede ser más descabellada.

En la traducción del artículo de Sklovski, "El arte como artificiox, y sin poner nota de ningún tipo, traduce nouvelle por relato. Transcribo como muestra un texto francés y su traducción: «A la fin de la nouvelle, le cheval est déjà tué, mais le mode de narration, le procédé n'est pas changé» $(T h L, 86) /$ "Al final del relato el caballo 
ya ha muerto, pero el modo de narración, el procedimiento, no cambia» (T. L., 63).

Solución distinta es la que adopta en la traducción del artículo de Sklovski, "La construcción de la "nouvelle" y de la novela". En esta ocasión incluye una nota diciendo que mantiene el término francés nouvelle (novela corta), pero que en muchas ocasiones podría haberse traducido por cuento (?) (T.L., 127). Y, en efecto, en todo el artículo se conserva el término francés nouvelle.

Otra solución es la que adopta, sin avisar, en la traducción del trabajo de B. Eichenbaum, Sobre la teoría de la prosa. Aquí traduce sistemáticamente nouvelle por cuento y no pone ninguna nota aclaratoria. Un ejemplo: "Le roman et la nouvelle ne sont pas des formes homogènes, mais au contraire des formes profondément étrangères l'une à l'autre» $(T h L, 202)$ / "La novela y el cuento no son formas homogéneas, sino, por el contrario, formas completamente extrañas una a otra" (T. L., 151). Y así en todo el artículo. No necesita comentario lo absurdo y erróneo de esta traducción, que lleva a un grave error conceptual -confusión de cuento y novela corta- que desvirtúa completamente el pensamiento del autor.

No acaba aquí, sin embargo, lo disparatado de las soluciones que adopta. En efecto, a la hora de traducir el trabajo siguiente de la antología, que pertenece también a B. Eichenbaum y lleva por título "Cómo está hecho "El Capote" de Gogol», pone una nota en la que advierte que sería mejor usar el término francés nouvelle o el italiano novella para caracterizar El Capote, aunque él utiliza el término cuento para tal caracterización. Pero no dice que en el texto francés se usa precisamente nouvelle (ThL, 212, y $T . L$., 159).

Pero es que todavía en la traducción del fragmento de B. Tomashevski que lleva por título "Temática", vuelve a traducir nouvelle por cuento, sin avisar nada. Véanse el texto francés y su traducción: «Dans le premier cas, nous avons affaire à des oeuvres "à sujet" (nouvelle, roman, poème épique)" (ThL, 267) / "En el primer caso se trata de obras "con argumento" (cuento, novela, poema épico)» (T. L., 202).

Creo que son suficientes estos ejemplos para desaconsejar vivamente la traducción castellana del texto básico del formalismo ruso a cualquiera que intente conocer el pensamiento de los formalistas sobre una cuestión tan importante como la tipología de los géneros narrativos. Quizá habría que ir más lejos y desaconsejarlo para conocer el pensamiento formalista en general. A las razones y defectos señalados quisiera añadir algún detalle más. Hay en la página 110 de la traducción una errata que hace incomprensible todo un epígrafe del artículo de O. Brik, "Ritmo y sintaxis». Donde pone El verso como unidad rítmica y semántica, debe sustituirse semán- 
tica por sintáctica, tal como dice el texto francés. Por último, uno de los términos empleados por Tinianov en su trabajo "Sobre la evolución literaria», creo que no ha sido correctamente traducido al castellano. Se trata del concepto de fonction synnome, que Tinianov opone a fonction autonome $(T h L, 123)$. Tanto la versión de este trabajo en $T$. L. (pág. 91) como la que se hace en Formalismo y vanguardia (pág. 121), traducen fonction synnome por función sinónima. Creo preferible la utilización del neologismo sínoma, lo mismo que synnome es neologismo en francés, y se evita la confusión con sinónima, pues el traductor francés no ha empleado synonyme tampoco. Además, autónomo y sínomo son compuestos de nomos y no de ónoma.

A la vista de todo lo anterior, la conclusión que se impone es clara: para conocer lo esencial de la teoría literaria de los formalistas rusos hay que prescindir de la versión castellana de la antología que tradujo y editó en francés T. Todorov. Pero también cabe la posibilidad de una nueva versión mucho más rigurosa. 